\title{
Tonemapping Multi-shot Digital Photographs of Real World Environments
}

\author{
Rehan Zia \\ Bournemouth University \\ The Media School \\ Dorset BH12 5BB, UK \\ rzia@bmth.ac.uk
}

\section{INTRODUCTION}

This paper explores different methods, techniques and workflow of tonemapping digital photographs of real world environments being employed by the researcher-practitioner, and, the merits and limitations associated with each. Three different real world environments are considered in order to test these methods and techniques under different lighting conditions, movement within the scene and the desired visual aesthetic of the researcherpractitioner.

\section{AIM}

The aim of this paper is to explore what the best practices for tonemapping photographs of real world environments for each of the three test case scenarios could be. This knowledge could then be incorporated into the researcher-practitioner's practice and utilised in the subsequent landscape photographs created.

\section{TEST CASES}

The three test case scenarios will comprise of a seascape, an image of Corfe Castle, and, an image of an urban environment. These three locations will provide three very distinct sets of challenges, i.e. the constant movement of the sea, the movement in the foliage around Corfe Castle, and, a very still environment with possibly people moving around respectively.

\section{TECHNIQUES \& WORKFLOW}

Each image would consist of multiple different exposures ranging from three to fifteen photographs shot at a two-stop interval by varying the shutter speed on a digital single lens reflex camera. A full-frame sensor camera is used in order to minimise the digital sensor noise. All images will be shot in camera RAW format in order to capture the maximum possible data.

Different methods of combining these exposures together will be explored ranging from fully automated tonemapping and exposure fusion in Photomatix to manual mask creation and layering in Adobe Photoshop.

Further enhancements may also be applied in Adobe Photoshop to test the tonal latitude of the image.

\section{LIMITATIONS}

Fully automated settings in tonemapping software such as Photomatix can create ghosting issues. Micro-contrast adjustments across the entire image can also introduce an illustration aesthetic which makes the image look unnatural. Semi-automatic options are available where the artist can highlight problematic areas suffering from ghosting issues.

Masking exposures in Adobe Photoshop and only revealing desired regions within each exposure can offer more control over larger image elements such as the land and the sky. However, it is difficult to make any micro-contrast adjustments using this method.

\section{FUTURE WORK}

Future work will look at merging these techniques in order to test different combinations of hybrid techniques by blending the resulting images together. 